\title{
Nano-buffer controlled electron tunneling to regulate heterojunctional interface emission
}

\author{
Wei Liu, Zhuxin Li, Zengliang Shi, Ru Wang, Yizhi Zhu and \\ Chunxiang $\mathrm{Xu}^{*}$
}

\begin{abstract}
Interface emission from heterojunction is a shortcoming for electroluminescent devices. A buffer layer introduced in the heterojunctional interfaces is a potential solution for the challenge. However, the dynamics for carrier tunneling to control the interface emission is still a mystery. Herein, the low-refractive $\mathrm{HfO}_{2}$ with a proper energy band configuration is employed as the buffer layer in achieving $\mathrm{ZnO}$-microwire/ $\mathrm{HfO}_{2} / \mathrm{GaN}$ heterojunctional light-emitting diodes (LEDs). The optically pumped lasing threshold and lifetime of the $\mathrm{ZnO}$ microwire are reduced with the introduced $\mathrm{HfO}_{2}$ layer. As a result, the interface emission is of blue-shift from visible wavelengths to $394 \mathrm{~nm}$ whereas the ultraviolet (UV) emission is enhanced. To regulate the interface recombination between electrons in the conduction band of $\mathrm{ZnO}$ and holes in the valence band of $\mathrm{GaN}$, the tunneling electrons with higher conduction band are employed to produce a higher tunneling current through regulation of thin $\mathrm{HfO}_{2}$ film causing blue shift and interface emission enhancement. Our results provide a method to control the tunneling electrons in heterojunction for high-performance LEDs.
\end{abstract}

Keywords: tunneling electron; light-emitting diode; heterojunctional interface; nano $\mathrm{HfO}_{2}$ buffer

Liu W, Li ZX, Shi ZL, Wang R, Zhu YZ et al. Nano-buffer controlled electron tunneling to regulate heterojunctional interface emission. Opto-Electron Adv 4, 200064 (2021).

\section{Introduction}

The heterojunction is one of the approaches to construct functional devices in semiconductors that are difficult to implant both $\mathrm{n}$ - and p-type conduction. However, undesired emission (interface emission) from electroluminescence (EL) usually happens at the interface due to the mismatched energy band configuration. Generally, a proper buffer layer was introduced to suppress the interface emission and improve the intrinsic emission ${ }^{1}$. In this case, the devices have to work at a higher turn-on voltage with higher energy consumption. Furthermore, the bottle-neck of p-doped $\mathrm{ZnO}$ has seriously impeded its desired application in short-wavelength optoelectronics ${ }^{2}$, especially as ultraviolet (UV) laser diodes ${ }^{3}$. Therefore,
$\mathrm{ZnO} / \mathrm{GaN}$ heterojunctions have been designed and the various layers have been introduced as the electron barrier layers, such as $\mathrm{Ga}_{2} \mathrm{O}_{3}{ }^{4}, \mathrm{ZnS}^{5}, \mathrm{MgO}^{6,7}, \mathrm{Al}_{1-x} \mathrm{Ga}_{x} \mathrm{~N}^{3}$, $\mathrm{AlN}^{8,9}, \mathrm{HfO}_{2}{ }^{10}, \mathrm{MgF}^{11}, \mathrm{Al}_{2} \mathrm{O}_{3}{ }^{12}, \mathrm{NiO}^{13}$ and $\mathrm{In}_{0.17} \mathrm{Al}_{0.83} \mathrm{~N}^{14}$. In these reports, the turn-on voltages have been elevated by increasing the thickness of electron barrier layers. It is expected to suppress the interface emission through insertion of a proper buffer layer with certain thickness $(\geq$ $20 \mathrm{~nm}$ ) for controlling the tunneling electrons or holes $^{12,13,15}$. In these situations, the UV emission of p-type $\mathrm{GaN}^{16,17}$ and interface emission were both reduced. However, is it possible to take advantage of the interface emission? How to control and utilize the interface emission still needs to be studied in the heterojunctional

State Key Laboratory of Bioelectronics, School of Biological Sciences \& Medical Engineering, Southeast University, Nanjing 210096, China. *Correspondence: CX Xu; E-mail: xcxseu@seu.edu.cn

Received: 7 August 2020; Accepted: 17 December 2020; Published: 25 September 2021; Available Online: 18 August 2021

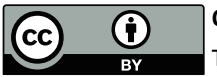

Open Access This article is licensed under a Creative Commons Attribution 4.0 International License.

To view a copy of this license, visit http://creativecommons.org/licenses/by/4.0/.

(C) The Author(s) 2021. Published by Institute of Optics and Electronics, Chinese Academy of Sciences. 
display field.

For the case of $\mathrm{ZnO} / \mathrm{GaN}$, another issue is the inevitable optical loss at the interface due to the similar refractive index of $\mathrm{GaN}$ and $\mathrm{ZnO}^{18}$. The insertion of buffer layer with a low refractive index is an effective way to improve the UV emission. For example, Zhu et al. ${ }^{18}$ introduced a PVK layer between $\mathrm{ZnO}$ micro-rod and $\mathrm{GaN}$ substrate and confined the optical field in $\mathrm{ZnO}$ cavity effectively. This was a prominent method to reduce optical loss and improve EL via insertion of a proper buffer layer.

In this work, a thin $\mathrm{HfO}_{2}$ film was introduced between $\mathrm{ZnO}$ and $\mathrm{GaN}$ to improve light reflectivity of interface and regulate tunneling electrons in the heterojunction. The influences of the thin $\mathrm{HfO}_{2}$ film on $\mathrm{PL}$ of $\mathrm{ZnO}$ microwire (MW) as well as $\mathrm{EL}$ of $\mathrm{ZnO} \mathrm{MW} / \mathrm{HfO}_{2} / \mathrm{GaN}$ light-emitting diodes (LEDs) were studied experimentally and theoretically. It was found that the improved UV emission of device with blue-shifted interface emission increased from $66.26 \%$ to $76.14 \%$ using $\mathrm{HfO}_{2}$ with the optimized thickness of $5 \mathrm{~nm}$. Moreover, the optical gain and loss were evaluated through whispering-gallery mode (WGM) lasing. Moreover, the electron tunneling dynamics was discussed based on the energy band configuration.

\section{Experiment}

\section{Material synthesis and device fabrication}

The n-type $\mathrm{ZnO}$ MWs were synthesized by using a chemical vapor deposition (CVD) method in our previous work ${ }^{19}$. To systematically study the buffer influence on the EL of the device, the $\mathrm{HfO}_{2}$ film was deposited on the $\mathrm{GaN}$ film by radio frequency magnetron sputtering with an $\mathrm{HfO}_{2}$ target (99.99\%). By moving the masks and changing the time of magnetron sputtering $(3,5$, and 7 min), a stepped $\mathrm{HfO}_{2}$ film was fabricated, and each step was named $\mathrm{GaN}$ 1, GaN 2, GaN 3 and GaN 4 respectively, as shown Fig. 1. The diagram of device fabrication is shown in Fig. 1. The individual $\mathrm{ZnO}$ MWs were placed on the steps for in-situ $\mu$-PL analysis, and then were bonded with ITO and GaN films to form ITO/ZnO/ $\mathrm{HfO}_{2} / \mathrm{GaN}$ LEDs separated by PMMA. Finally, the ITO/ZnO/ $/ \mathrm{HfO}_{2} / \mathrm{GaN}$ LEDs constructed by ITO bonded $\mathrm{ZnO} \mathrm{MW}$ are named as LED 1, LED 2, LED 3, and LED 4, respectively.

Instruments

The atomic force microscope (AFM) images were meas-

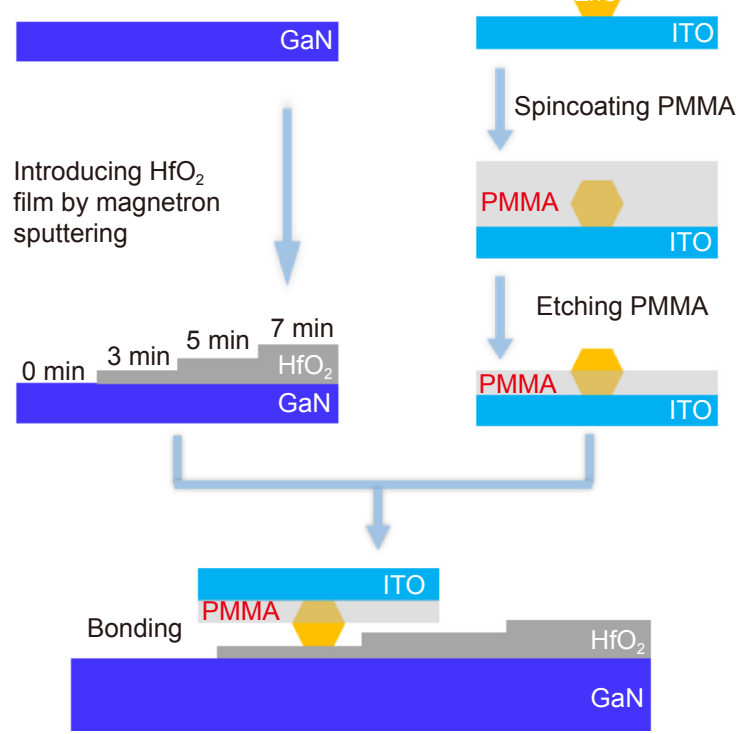

Fig. 1 | The diagram of device fabrication.

ured in tapping mode by Brukers Dimension Icon Atomic Force Microscopy under ambient conditions. Optically pumped spectra of the device were measured by a home-made confocal microphotoluminescence ( $\mu$-PL) setup (Olympus BX35) excited by a $325 \mathrm{~nm}$ femtosecond pulsed laser with a $10 \times$ objective. The EL and I-V characteristics of the samples were conducted in a cooling-heating stage (INSTEC mk2000), with the $\mu$-PL setup and a semiconductor characterization system (Keithley 4200). The PL and EL spectra were all collected through a spectrometer (Princeton Instruments Acton SP2500i). Time-resolved PL (TR-PL) was measured by a streak camera (Optronis Optoscope sc-10) and a femtosecond pulsed laser (100 fs pulsed duration and $1 \mathrm{kHz}$ repetition rate) as signal detector and excitation source, respectively.

\section{Simulations}

The proposed structure is analyzed using a finite element method (FEM). The reflectivity of interface in these structures is simulated. In the simulation, the refractive indices of the $\mathrm{ZnO}, \mathrm{HfO}_{2}$ and $\mathrm{GaN}$ were taken to be 2.5, 1.98 and 2.3, respectively. The thicknesses of the $\mathrm{ZnO}$ layer and $\mathrm{GaN}$ layer were both set to be $2.5 \mu \mathrm{m}$. The $\mathrm{HfO}_{2}$ layer was inserted between the $\mathrm{ZnO}$ layer and $\mathrm{GaN}$ layer, where the thicknesses were $0,5.03,8.79$ and 12.55 $\mathrm{nm}$. The light is perpendicularly emitted from the $\mathrm{ZnO}$ at the wavelength of $390 \mathrm{~nm}$.

Quantum size effects are not significant in the $\mathrm{ZnO}$ MW, so a $1 \mathrm{D}$ semi-empirical method can be used in the 
simulation. As displayed on the inset of Fig. 7(b), the thickness of the device was $1 \mu \mathrm{m}$ with an n-type doping concentration of $1 \times 10^{16} \mathrm{~cm}^{-3}$ for $\mathrm{ZnO}$ and a p-type doping concentration of $1 \times 10^{17} \mathrm{~cm}^{-3}$ for $\mathrm{GaN}$, in which the thickness of insulating layer was adjustable. The driving voltage was $40 \mathrm{~V}$. To illustrate the basic physics simply, the electrodes at the ends of the device were assumed to be Ohmic contacts and the Dirichlet boundary conditions were applied for the carrier concentration and electrical potential. The thermionic emission model to de- termine the current transfer occurring between the different materials and Shockley-Read-Hall recombination feature were considered as well. The distributions of conduction band and valence band were analyzed near the $\mathrm{ZnO}-\mathrm{HfO}_{2}-\mathrm{GaN}$ junctions, which is modulated by the thickness of $\mathrm{HfO}_{2}$.

\section{Results and discussion}

The schematic diagram and EL spectra for $\mathrm{ZnO} / \mathrm{GaN}$ LED are shown in Fig. 2(a) and 2(b), respectively.
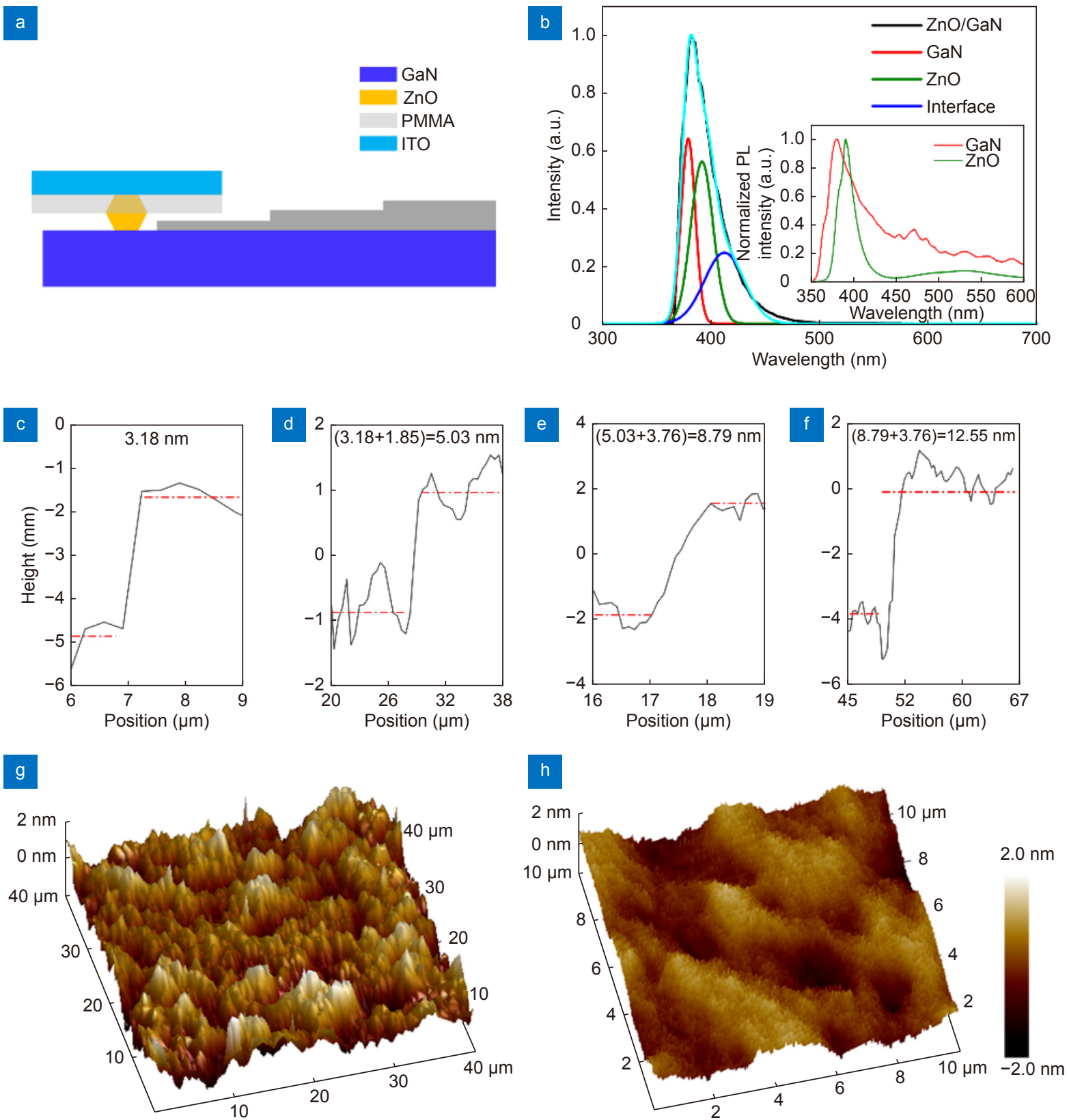

Fig. 2 | (a) A schematic diagram for ZnO/GaN LEDs. (b) The Gaussian decomposition of EL spectra for LED and the inset depicts the PL spectra of the GaN film as well as $\mathrm{ZnO}$ MWs. (c-f) The height of the step-like $\mathrm{HfO}_{2}$ film with different sputtering time (c) 2 min, (d) 3 min, (e) 5 min and (f) $7 \mathrm{~min}$. (g, h) AFM image for the GaN surface (g) before and (h) after bonding $\mathrm{HfO}_{2}$ film with thickness of $5.03 \mathrm{~nm}$. 
Moreover, the peak of PL spectra for $\mathrm{ZnO} \mathrm{MW}$ and GaN film at $391 \mathrm{~nm}$ and $379 \mathrm{~nm}$ are demonstrated in the inset of Fig. 2(b). The EL spectrum of $\mathrm{ZnO} / \mathrm{GaN}$ LED includes three parts: peaks at $379 \mathrm{~nm}$ from GaN, $391 \mathrm{~nm}$ from $\mathrm{ZnO}$ and $414 \mathrm{~nm}$ from the interface emission, respectively. The $\mathrm{HfO}_{2}$ film was tested by a stepped structure shown in Fig. 3(a) using AFM. The thickness of the $\mathrm{HfO}_{2}$ film in $\mathrm{GaN} 2$ is $5.03 \mathrm{~nm}, \mathrm{GaN} 3$ is $8.79 \mathrm{~nm}$ and $\mathrm{GaN} 4$ is $12.55 \mathrm{~nm}$ as shown in Fig. 2(c)-2(f). The AFM image of GaN film in Fig. 2(g) exhibits a smooth surface with the average roughness of $\sim 0.551 \mathrm{~nm}$. The morphology of $\mathrm{GaN}$ film became smoother with the average roughness of $\sim 0.337 \mathrm{~nm}$ after the modification by $\mathrm{HfO}_{2}$, as shown in Fig. 2(h). It can be observed that the interface contact can be improved after the GaN film bonded with $\mathrm{HfO}_{2}$.

In order to investigate the influence of $\mathrm{HfO}_{2}$ thickness on optical gain and loss of $\mathrm{ZnO} \mathrm{MW} / \mathrm{HfO}_{2} / \mathrm{GaN}$ heterjunctions, the in-situ PL experiments were carried out and the obtained lasing spectra for $\mathrm{ZnO} \mathrm{MW}$ on GaN films with different excitation power densities are displayed in Fig. 3. All samples demonstrated the WGM behaviors and the lasing characteristics, i.e., lasing threshold, intensity, and position, are modulated depending on the variations in the modified film at the boundaries of the cavity.

Although the lasing modes were located at the same position, the number of modes is increased from 9 to 11 at the same excitation power of $20.4 \mu \mathrm{W}$, as shown in Fig. 3(c) and 3(d). In addition, the lasing was enhanced at the same excitation power and lasing thresholds of $\mathrm{ZnO} \mathrm{MW}$ on GaN 2, 3 and 4 were lower than that of GaN 1 as shown in Fig. 3(b). In order to understand the reason, the reflectivity at $390 \mathrm{~nm}$ was simulated by a multi-physical field method. It can be seen that the reflectivity of $390 \mathrm{~nm}$ was increased $(0.129,0.132,0.140$, 0.153 and 0.283 ) with the film thickness increasing. The thresholds of $\mathrm{ZnO}-\mathrm{MW}$ lasing were decreased whereas the quality of microcavity is increased by the $\mathrm{HfO}_{2}$ modified GaN film.

For more insights into dynamic coupling processes, the time-resolved photoluminescence (TRPL) spectra were recorded at room temperature, as shown in Fig. 4. The normalized TRPL decay curves of the $\mathrm{ZnO} \mathrm{MW}$ on GaN 1, GaN 2, GaN 3, and GaN 4 (the detailed definition is in Experiment part) were well-fitted by the

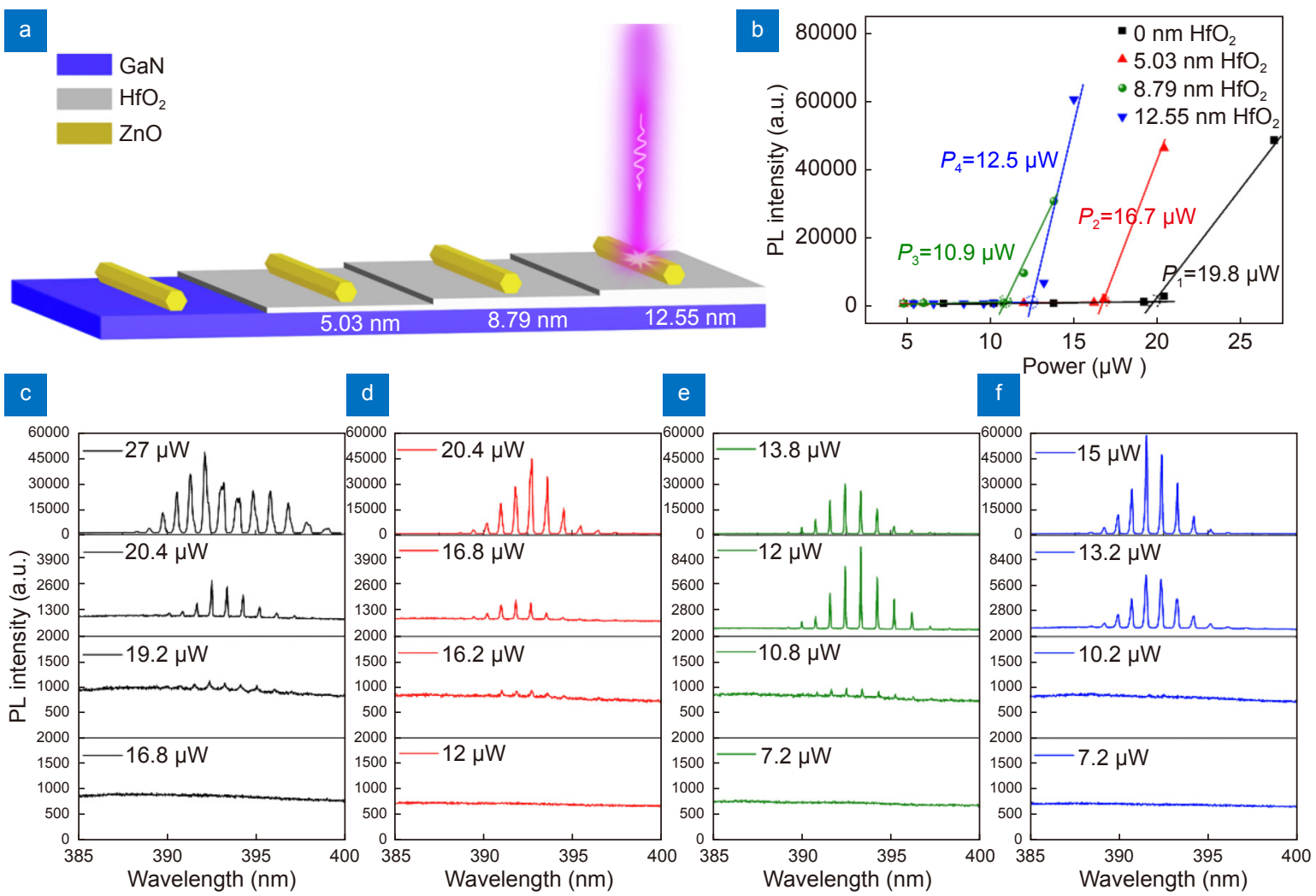

Fig. 3 | (a) Schematic diagram of $\mathrm{ZnO} / \mathrm{HfO}_{2} / \mathrm{GaN}$ for in-situ optical test. (b) Lasing emission intensity for $\mathrm{ZnO} \mathrm{MW}$ versus the excitation power density on different substrates. (c-f) the corresponding PL spectra under different excitation power densities for ZnO MW with different thickness of $\mathrm{HfO}_{2}$ films: (c) $0 \mathrm{~nm}$, (d) $5.03 \mathrm{~nm}$, (e) $8.79 \mathrm{~nm}$ and (f) $12.55 \mathrm{~nm}$. 


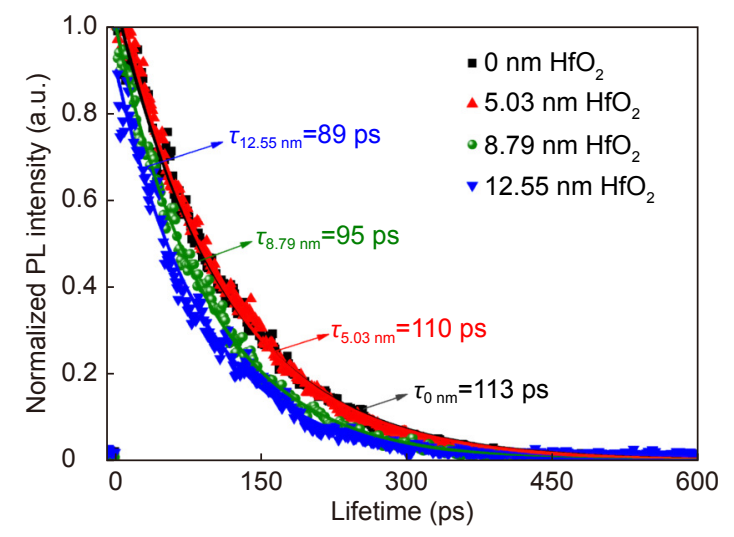

Fig. 4 | The lifetime of $\mathrm{ZnO} M W$ on various substrates with excitation power of $8 \mu \mathrm{W}$.

mono-exponential function. The decay lifetime $e^{19-21}$ is defined as follows:

$$
I(t)=I_{0} \exp \left(-t / \tau_{\text {photonic }}\right),
$$

here, $I_{0}$ is the starting PL intensity before the decay. The reduction for the lifetime of $\mathrm{ZnO} \mathrm{MW}$ from 113 ps to 89 ps after bonding the $\mathrm{HfO}_{2}$ film is attributed to the aggravation of non-radiative transition. The PL intensity of $\mathrm{ZnO} \mathrm{MW}$ was therefore enhanced after the $\mathrm{HfO}_{2}$ film modification, which is in agreement with the results of in-suit PL experiments as depicted in Fig. 3. Due to the inserted $\mathrm{HfO}_{2}$ buffer layer with low refractive index, the reflectivity of GaN film was increased to improve the Q factor of the $\mathrm{ZnO} \mathrm{MW}$ microcavity. The laser threshold was hence decreased by the increasing of the thickness of the $\mathrm{HfO}_{2}$ film. After the interface was modified by the $\mathrm{HfO}_{2}$ film, the reflectivity was enhanced and roughness was reduced. The Purcell effect is therefore strengthened.

A schematic diagram of $\mathrm{ZnO} / \mathrm{HfO}_{2} / \mathrm{GaN}$ LED and I-V curves of these LEDs are depicted in Fig. 5(a). After the $\mathrm{HfO}_{2}$ film was sputtered on the interface of the $\mathrm{GaN}$ film, the I-V curve of the devices displayed a better rectification property. The turn-on voltage was increased with the $\mathrm{HfO}_{2}$ film thickening. Although the driving voltage increases to $40 \mathrm{~V}$, the device with $60 \mathrm{~nm} \mathrm{HfO}_{2}$ film still had no current produced (Open circuit state). The normalized EL spectra of the LEDs under a driving current of $1 \mathrm{~mA}$ are shown in Fig. 5(b). It was observed that the blue shift of the EL spectra occurred after 5.03 $\mathrm{nm} \mathrm{HfO}_{2}$ film bonding. Meanwhile, the spectrum of the device was the bluest in chromaticity coordinates (CIE 1931) in Fig. 5(g). The EL spectra and light pictures of the devices are shown in Fig. 5(c)-5(f). The light picture shows a same bluish violet after $\mathrm{HfO}_{2}$ film bonding. The device with 5.03-nm-thick $\mathrm{HfO}_{2}$ film had the highest brightness light emission under the same current as shown in Fig. 5(h). It was attributed to high electron tunneling efficiency of the device with $5.03 \mathrm{~nm} \mathrm{HfO}_{2}$ film and the Q-factor enhancement on the microcavity of $\mathrm{ZnO}$ MW. It should be noted that the devices with $\mathrm{HfO}_{2}$ films (more than $5.03 \mathrm{~nm}$ ) had the lower brightness than that of the device without $\mathrm{HfO}_{2}$ film at the same current. It was attributed to the fact that more electrons were lost non-radiatively during the electron tunneling process in the thicker $\mathrm{HfO}_{2}$ film.

In order to understand the change in origin of EL spectrum of the device, the EL of devices at the current of $1 \mathrm{~mA}$ was analyzed by a Gaussian method as shown in Fig. 6(a-d). The EL spectra of LEDs demonstrated three peaks which were centered at $\sim 379 \mathrm{~nm}, \sim 390 \mathrm{~nm}$ and $\sim 410 \mathrm{~nm}$, respectively. In Fig. 6(a)-6(d), it can be observed that the Gaussian curves fitted well with the experimental curves. In consideration of the PL spectra for $\mathrm{GaN}$ with $\mathrm{ZnO}$, the $\mathrm{UV}$ emission bands were centered at $379 \mathrm{~nm}$ and $390 \mathrm{~nm}$ related to near-bandedge recombination of $\mathrm{GaN}$ and $\mathrm{ZnO}$, whereas the emission band at $\sim 410 \mathrm{~nm}$ was corresponding to the recombination of tunneling electrons in $\mathrm{ZnO}$ and the holes in $\mathrm{GaN}^{1,22}$. The location of EL emission peak of the recombination in $\mathrm{GaN}$ and $\mathrm{ZnO}$ was varied slightly with the change of the thickness of $\mathrm{HfO}_{2}$ film, while the interface emission by the recombination of tunneling electrons of $\mathrm{ZnO}$ and holes in $\mathrm{GaN}$ was located from $394 \mathrm{~nm}$ to 416 nm, respectively. For LED 1, LED 2, LED 3 and LED 4, the emission peaks were located at $\sim 412 \mathrm{~nm}, \sim 394 \mathrm{~nm}$, $\sim 416 \mathrm{~nm}$ and $\sim 414 \mathrm{~nm}$. To understand the UV content of the devices, the integral intensities of UV $(<400 \mathrm{~nm})$ and visible spectra ( $>400 \mathrm{~nm}$ and $<700 \mathrm{~nm}$ ) were calculated as shown in Fig. 6(a)-6(d). From the insets of Fig. 6(a)-6(d), it can be observed that the UV light content of LED 1, LED 2, LED 3, and LED 4 are 66.26\%, 76.14\%, $61.30 \%$ and $58.61 \%$, respectively. Interestingly, LED 2 has high-content UV light emission with high brightness. The peak positions and full width at half maxima (FWHM) of emissions are displayed in Fig. 6(e) and 6(f). All the emission peak positions are blue shifted. Especially, the interface emission is become narrow and blue shifted from $414 \mathrm{~nm}$ to $394 \mathrm{~nm}$ after introducing the 5.03 nm $\mathrm{HfO}_{2}$ film.

The p-type GaN in our work had a strong UV emission peak at $379 \mathrm{~nm}$ as shown in Fig. 6(a) to 6(d), which is well matched with the previous reports ${ }^{16,17}$. The hole carrier concentration of GaN film is $3.0 \times 10^{17} \mathrm{~cm}^{-3}$. The 
a

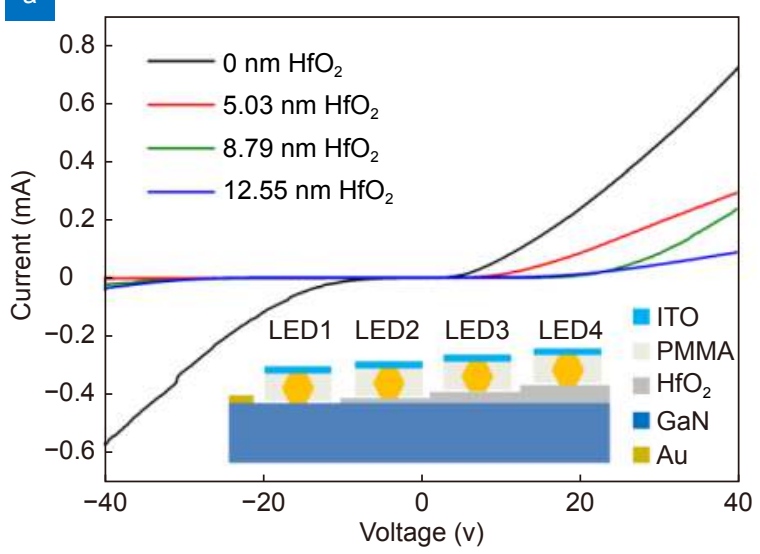

b

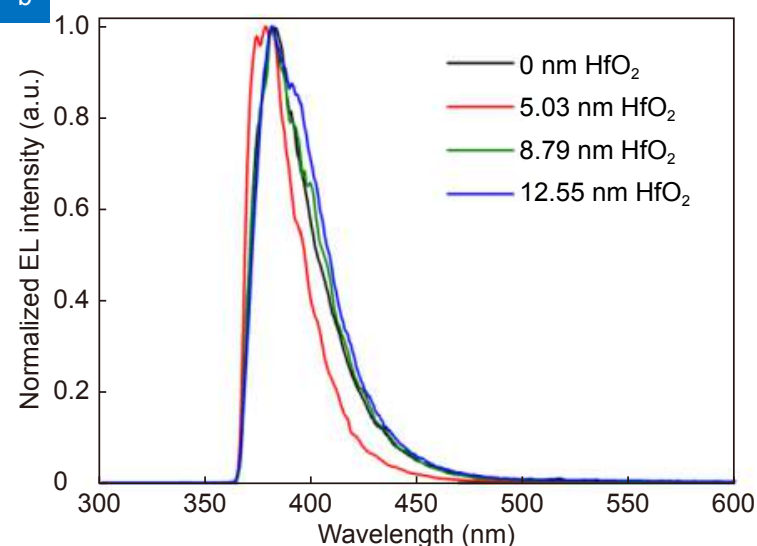

e

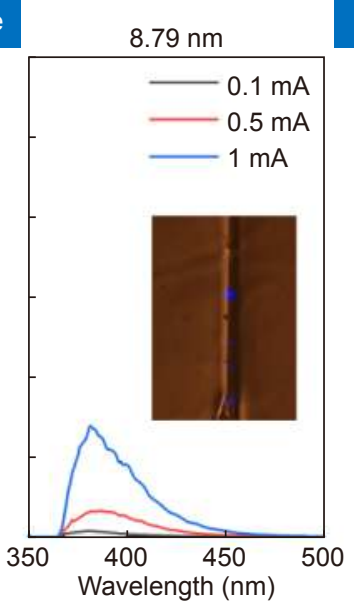

$\mathrm{f}$

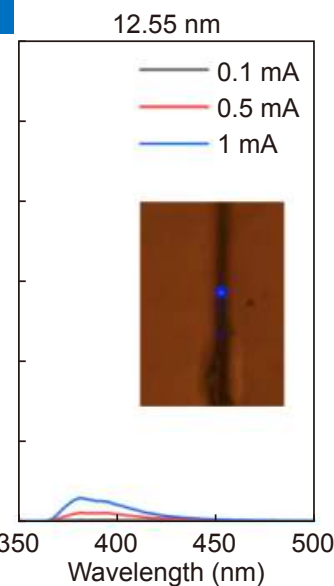

g

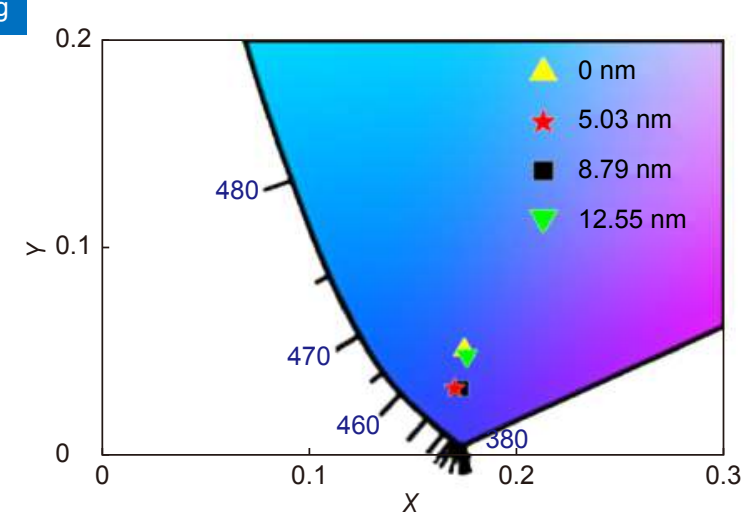

h

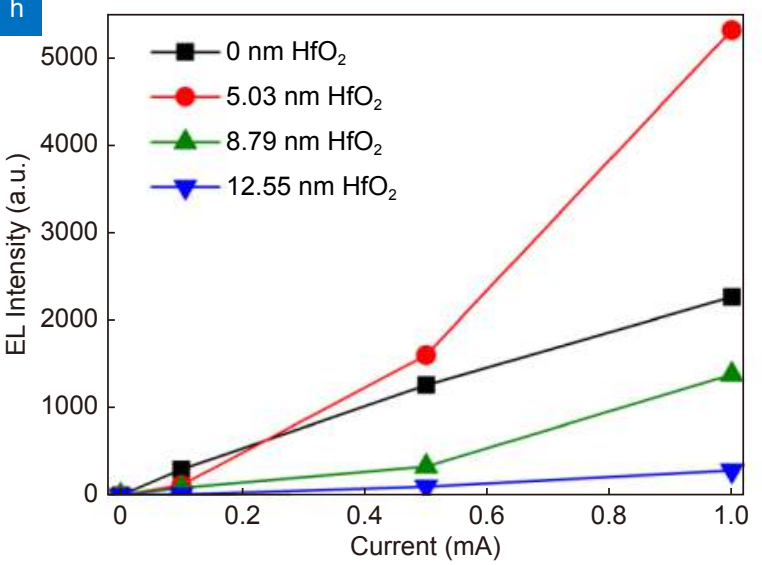

Fig. 5 | (a) I-V characteristics of ITO/ZnO/HfO $/ \mathrm{GaN}$ LEDs. The inset is a schematic diagram for ITO/ZnO/HfO $/ \mathrm{GaN}$ LEDs. (b) Normalized EL spectra for LEDs under an excitation current of $1 \mathrm{~mA}$. (c-f) EL intensity of $\mathrm{ZnO} / \mathrm{HfO}_{2} / \mathrm{GaN} \mathrm{LEDs} \mathrm{with} \mathrm{HfO}_{2}$ films of different thickness and the inset is the light pictures: (c) $0 \mathrm{~nm}$, (d) $5.03 \mathrm{~nm}$, (e) $8.79 \mathrm{~nm}$, (f) $12.55 \mathrm{~nm}$. (g) Chromaticity coordinates of the spectra in (b). (h) EL peak intensities of the LEDs from (c) to $(f)$.

PL spectrum shown in Fig. 2(b) was in agreement with the results of EL spectra of $\mathrm{GaN}$ and $\mathrm{ZnO}$ in the LEDs. The energy level position changes of interface on the junction were influenced by the thickness of $\mathrm{HfO}_{2}$ film as simulated in Fig. 7(a). Serious band bending phenomenon occurred after introducing $\mathrm{HfO}_{2}$ buffer layer indicat- ing higher level tunneling electron-hole recombination after bonding $\mathrm{HfO}_{2}$ film. When the thickness of buffer layer was increased, the band bending became smooth, resulting in blue shift from recombination between tunneling electrons of $\mathrm{ZnO}$ and holes of $\mathrm{GaN}$ after bonding thin $\mathrm{HfO}_{2}$ film. To systematically investigate the migration 

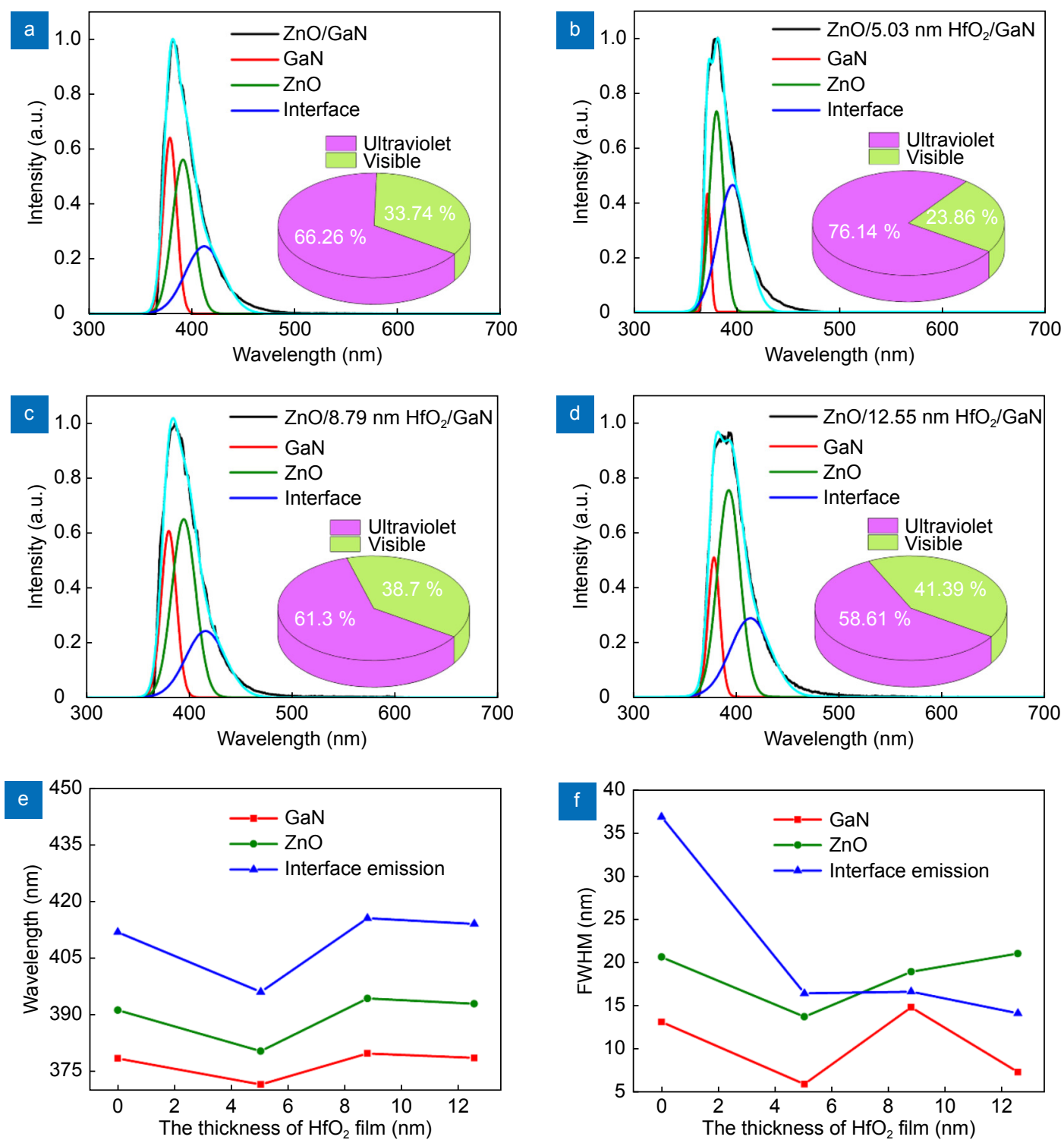

Fig. 6 | Gaussian conversion of Fig. 5(c)-5(f) at a current of 1 mA: (a) 0 nm, (b) $5.03 \mathrm{~nm}$, (c) $8.79 \mathrm{~nm}$, (d) $12.55 \mathrm{~nm}$, and the inset images depict the contents of UV and visible lights. (e) The peak positions of emissions and (f) FWHM of emissions for these LEDs.

of electron in heterojunction, the distributions of electronic current influenced by different thicknesses of $\mathrm{HfO}_{2}$ film were simulated using the finite element method. The results are shown in Fig. 7(b) with a band structure diagram. The electrons were gathered at the highest electronic current density and formed the tunneling current of the device with the $5.03 \mathrm{~nm} \mathrm{HfO}_{2}$ film.

Schematic band structures based on theoretical simulation of transportations of the electrons and holes in these structures are illustrated in Fig. 8(a)-8(c). Different from previous reports ${ }^{10}$, the electrons in these devices with different thicknesses of $\mathrm{HfO}_{2}$ films tunneled through $\mathrm{HfO}_{2}$ film in the junction as shown in Fig. 8(b) and 8(c). In Fig. 8(b), it can be observed that the elec- trons tunneled through the thin $\mathrm{HfO}_{2}$ film $(5.03 \mathrm{~nm})$ and a part of electrons produced in $\mathrm{ZnO}$ was gathered at higher conduction band. Then they tunneled into valence band of $\mathrm{GaN}$ based on theoretical simulation as shown in Fig. 8(b). This was the reason for the blue shift $(394 \mathrm{~nm})$ at the interface between $\mathrm{ZnO}$ and GaN. From Fig. 8(c), it was noted that the electrons were difficult to tunnel through the thick $\mathrm{HfO}_{2}$ film and only a small part of electrons tunneled into valence band of $\mathrm{GaN}$ because of energy losses. The main reason was the recombination at the interface between $\mathrm{ZnO}$ and $\mathrm{GaN}$ with a red shift $(414 \mathrm{~nm})$ after a bonding of thicker $\mathrm{HfO}_{2}$ film. The device modified with the thin $\mathrm{HfO}_{2}$ film regulated the interface emission from visible to ultraviolet through 


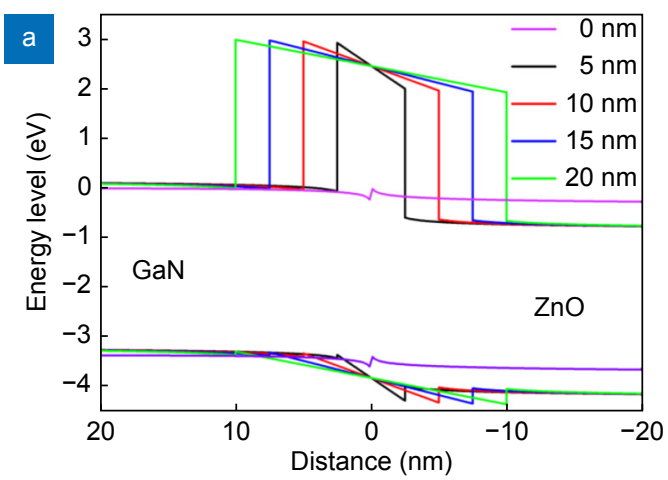

Fig. 7 | (a) Energy band of $\mathrm{ZnO} / \mathrm{HfO}_{2} / \mathrm{GaN}$ LEDs based on theoretical simulation. $\mathrm{ZnO} / \mathrm{HfO}_{2} / \mathrm{GaN}$ LEDs. The inset picture displays a structure diagram of simulation.
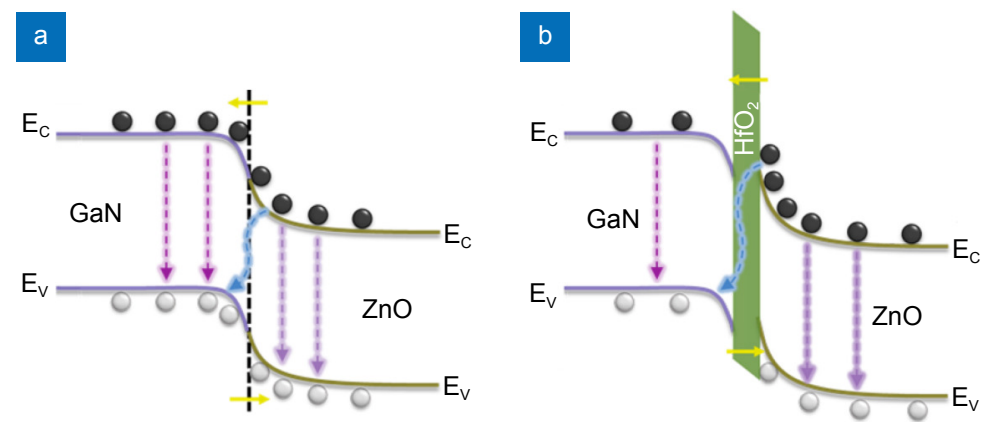
Fig. 8 | Schematic band structures of (a) $\mathrm{ZnO} / \mathrm{GaN}$, (b) $\mathrm{ZnO} /$ thin $\mathrm{HfO}_{2} / \mathrm{GaN}$ and (c) $\mathrm{ZnO} /$ thick $\mathrm{HfO}_{2} / \mathrm{GaN}$.

controlling the electron tunneling process and produced a higher brightness UV light.

\section{Conclusions}

In summary, $\mathrm{ZnO} / \mathrm{HfO}_{2} / \mathrm{GaN}$ UV LEDs were fabricated, and the tunneling electrons as well as optical losses in the LED were regulated though a nano $\mathrm{HfO}_{2}$ layer. The PL spectra of $\mathrm{ZnO} \mathrm{MW}$ were enhanced and threshold of the laser was decreased (from $19.8 \mu \mathrm{W}$ to $16.7 \mu \mathrm{W}$ ) at the same time based on in-situ PL experiment. Considering the device, the tunneling electrons with higher conduction band produced a higher tunneling current which is regulated by the thin $\mathrm{HfO}_{2}$ film. As a result, the peak position of interface emission by the recombination of the tunneling electrons in $\mathrm{ZnO}$ and holes in $\mathrm{GaN}$ had a blue shift (from $414 \mathrm{~nm}$ to $394 \mathrm{~nm}$ ) with increment in content of UV light ( $<400 \mathrm{~nm}$ ) (from $66.26 \%$ to $76.14 \%$ ). Over all, the interface emission of LED was regulated from visible to ultraviolet via insertion of a thin $\mathrm{HfO}_{2}$ buffer layer. The method provided a way to improve the performance of UV LEDs and regulate the tunneling electrons in heterojunction for illumination, display, and medical fields.

\section{References}

1. You DT, Xu CX, Qin FF, Zhu Z, Manohari AG et al. Interface
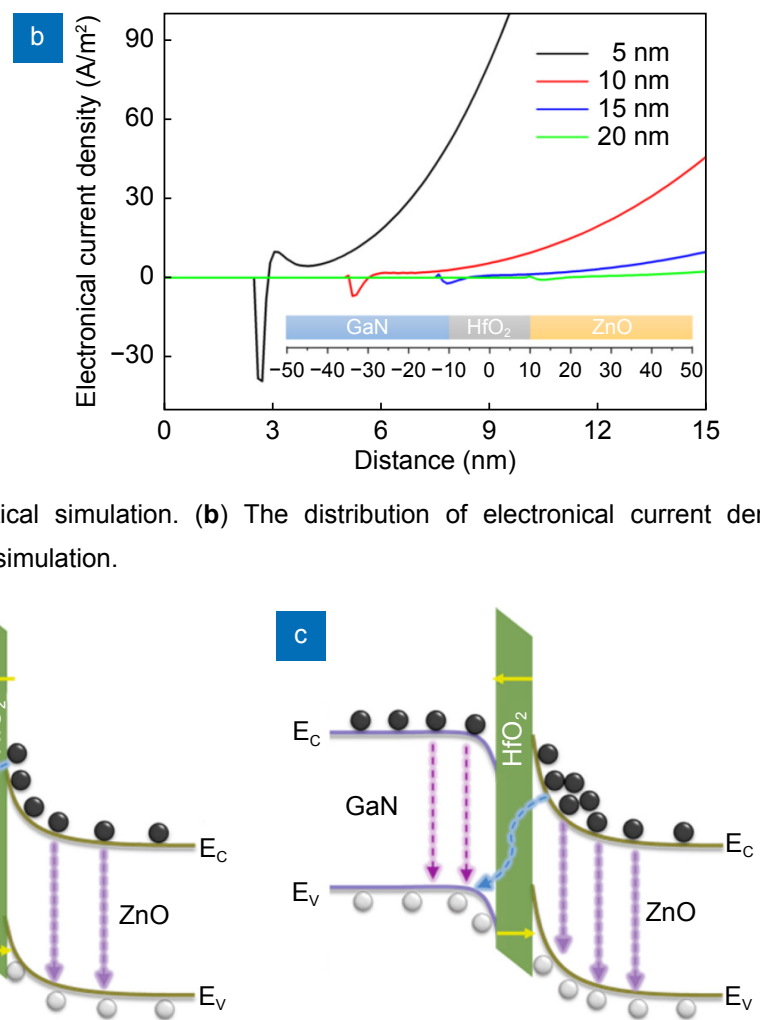

(b) The distribution of electronical current density in 
10. Huang $\mathrm{HH}$, Fang GJ, Mo XM, Long $\mathrm{H}$, Yuan LY et al. ZnObased fairly pure ultraviolet light-emitting diodes with a low operation voltage. IEEE Electron Device Lett 30, 1063-1065 (2009).

11. Jeong $\mathrm{S}, \mathrm{Kim} \mathrm{H}$. High light output efficiency of $\mathrm{n}-\mathrm{ZnO} / \mathrm{p}-\mathrm{GaN}$ heterojunction light-emitting diodes fabricated with a $\mathrm{MgF}_{2}$ electron-blocking layer. App/ Phys Express 9, 015501 (2016).

12. Yang L, Liu WZ, Xu HY, Ma JG, Zhang $C$ et al. Enhanced near-UV electroluminescence from $\mathrm{p}-\mathrm{GaN} / \mathrm{i}-\mathrm{Al}_{2} \mathrm{O}_{3} / \mathrm{n}-\mathrm{ZnO}$ heterojunction LEDs by optimizing the insulator thickness and introducing surface plasmons of Ag nanowires. J Mater Chem C 5, 3288-3295 (2017).

13. Wang $\mathrm{H}$, Zhao $\mathrm{Y}, \mathrm{Wu} \mathrm{C}$, Dong $\mathrm{X}$, Zhang $\mathrm{BL}$ et al. Ultraviolet electroluminescence from $\mathrm{n}-\mathrm{ZnO} / \mathrm{NiO} / \mathrm{p}-\mathrm{GaN}$ light-emitting diode fabricated by MOCVD. J Lumin 158, 6-10 (2015).

14. Wang X, Gan XW, Zhang GZ, Su X, Zheng MJ et al. The function of an $\ln _{0.17} \mathrm{Al}_{0.83} \mathrm{~N}$ interlayer in $\mathrm{n}-\mathrm{ZnO} / \mathrm{In}_{0.17} \mathrm{Al}_{0.83} \mathrm{~N} / \mathrm{p}-\mathrm{GaN}$ heterojunctions. App/ Surf Sci 393, 221-224 (2017).

15. Mo XM, Long $\mathrm{H}$, Wang HN, Li SZ, Chen $\mathrm{Z}$ et al. Enhanced ultraviolet electroluminescence and spectral narrowing from $\mathrm{ZnO}$ quantum dots/GaN heterojunction diodes by using high- $k \mathrm{HfO}_{2}$ electron blocking layer. Appl Phys Lett 105, 063505 (2014).

16. Huang $\mathrm{HH}$, Fang GJ, Li Y, Li SZ, Mo XM et al. Improved and color tunable electroluminescence from $n-\mathrm{ZnO} / \mathrm{HfO}_{2} / \mathrm{p}-\mathrm{GaN}$ heterojunction light emitting diodes. Appl Phys Lett 100, 233502 (2012).

17. Zhang XM, Lu MY, Zhang Y, Chen LJ, Wang ZL. Fabrication of a high-brightness blue-light-emitting diode using a $\mathrm{ZnO}$ nanowire array grown on $\mathrm{p}-\mathrm{GaN}$ thin film. Adv Mater 21, 2767-2770 (2009).
18. Zhu GY, Xu CX, Cai LS, Li JT, Shi ZL et al. Lasing behavior modulation for $\mathrm{ZnO}$ whispering-gallery microcavities. ACS Appl Mater Interfaces 4, 6195-6201 (2012).

19. Lu JF, Jiang MM, Wei M, Xu CX, Wang SF et al. Plasmon-induced accelerated exciton recombination dynamics in $\mathrm{ZnO} / \mathrm{Ag}$ hybrid nanolasers. ACS Photonics 4, 2419-2424 (2017).

20. Lu JF, Shi ZL, Wang YY, Lin Y, Zhu QX et al. Plasmon-enhanced Electrically Light-emitting from ZnO Nanorod Arrays/pGaN Heterostructure Devices. Sci Rep 6, 25645 (2016).

21. Oulton RF. Surface Plasmon lasers: sources of nanoscopic light. Mater Today 15, 26-34 (2012).

22. Liu W, Li ZX, Shi ZL, Chen F, Zhu YZ et al. Symmetrical bi-heterojunction alternating current ultraviolet light-emitting diode. IEEE Electron Device Lett 41, 252-255 (2020).

\section{Acknowledgements}

This work was supported by NSFC (11734005, 62075041, 61821002), National Key Research and Development Program of China (2018YFA0209101, 2017YFA0700500).

\section{Author contributions}

All authors commented on the manuscript. C. X. Xu proposed the original idea and supervised the project. W. Liu, Z. X. Li and Z. L. Shi fabricated the samples and performed the measurements. W. Liu wrote the manuscript. And W. Liu, R. Wang and Y. Z. Zhu did the lasing experiment. W. Liu, Z. X. $\mathrm{Li}$ and Z. L. Shi contributed equally to this work.

\section{Competing interests}

The authors declare no competing financial interests. 